\title{
A research synthesis of therapeutic interventions for whiplash-associated disorder: Part 1 - overview and summary
}

\author{
Robert W Teasell $\mathrm{MD}^{1,2,3}$, J Andrew McClure BA ${ }^{1}$, David Walton PhD candidate ${ }^{4}$, Jason Pretty BA ${ }^{1}$, \\ Katherine Salter $B A^{1}$, Matthew Meyer BA ${ }^{1}$, Keith Sequeira $M^{2}{ }^{2}$, Barry Death MD $^{2}$
}

\begin{abstract}
RW Teasell, JA McClure, D Walton, et al. A research synthesis of therapeutic interventions for whiplash-associated disorder: Part 1 overview and summary. Pain Res Manage 2010;15(5):287-294.
\end{abstract}

Whiplash-associated disorder (WAD) represents a significant public health problem, resulting in a substantial socioeconomic burden throughout the industrialized world, wherever costs are documented. While many treatments have been advocated for patients with WAD, scientific evidence of their effectiveness is often lacking. A systematic review was conducted to evaluate the strength of evidence supporting various WAD therapies. Multiple databases (including Web of Science, EMBASE and PubMed) were searched to identify all studies published from January 1980 through March 2009 that evaluated the effectiveness of any clearly defined treatment for acute (less than two weeks), subacute (two to 12 weeks) or chronic (longer than 12 weeks) WAD. The present article, the first in a five-part series, provides an overview of the review methodology as well as a summary and discussion of the review's main findings. Eighty-three studies met the inclusion criteria, 40 of which were randomized controlled trials. The majority of studies $(n=47)$ evaluated treatments initiated in the chronic stage of the disorder, while 23 evaluated treatments for acute WAD and 13 assessed therapies for subacute WAD. Exercise and mobilization programs for acute and chronic WAD had the strongest supporting evidence, although many questions remain regarding the relative effectiveness of various protocols. At present, there is insufficient evidence to support any treatment for subacute WAD. For patients with chronic WAD who do not respond to conventional treatments, it appears that radiofrequency neurotomy may be the most effective treatment option. The present review found a relatively weak but growing research base on which one could make recommendations for patients at any stage of the WAD continuum. Further research is needed to determine which treatments are most effective at reducing the disabling symptoms associated with WAD.

Key Words: Evidence-based medicine; Exercise; Neck pain; Randomized controlled trials; Whiplash-associated disorder

\section{Une synthèse de la recherche sur les interventions thérapeutiques à l'égard des troubles liés aux coups de fouet cervicaux (TCFC) : Partie 1 - Aperçu et résumé}

Les troubles liés aux coups de fouet cervicaux (TCFC) représentent un problème important en santé publique, associé à des coûts sociaux et économiques substantiels dans le monde industrialisé. De nombreux traitements sont préconisés pour les patients ayant des TCFC, mais souvent, on ne possède pas de données scientifiques probantes en étayant l'efficacité. Les chercheurs ont procédé à une analyse systématique pour évaluer la qualité des preuves associées aux diverses thérapies des TCFC. Ils ont effectué des recherches dans de multiples bases de données (y compris Web of Science, EMBASE et PubMed) pour repérer toutes les études publiées entre janvier 1980 et mars 2009 qui évaluaient l'efficacité de tout traitement clairement défini en cas de TCFC aigu (moins de deux semaines), subaigu (de deux à 12 semaines) ou chronique (plus de 12 semaines). Le présent article, premier d'une série de cinq, contient un aperçu de la méthodologie d'analyse ainsi qu'un résumé et un exposé des principaux résultats de l'analyse. Quatre-vingt-trois études respectaient les critères d'inclusion, dont 40 étaient des essais aléatoires et contrôlés. La majorité des études ( $\mathrm{n}=47)$ évaluaient des traitements amorcés pendant la phase chronique, tandis que 23 évaluaient des traitements des TCFC aigus et 13, des thérapies des TCFC subaigus. Les programmes d'exercice et de mobilisation pour les TCFC aigus et chroniques s'associaient aux données justificatives les plus solides, même si de nombreuses questions demeurent quant à l'efficacité relative de divers protocoles. Pour l'instant, les données probantes sont insuffisantes pour soutenir un quelconque traitement des TCFC subaigus. Chez les patients ayant un TCFC chronique qui ne répondent pas aux traitements classiques, il semble que la neurotomie par radiofréquence constituerait la solution thérapeutique la plus efficace. La présente analyse a permis de dépister un ensemble de recherches relativement faible, mais croissant à partir duquel il est possible de faire des recommandations aux patients à toutes les phases du continuum des TCFC. D'autres recherches s'imposent pour déterminer les traitements les plus efficaces à réduire les symptômes invalidants associés aux TCFC.
A s a significant public health problem and socioeconomic burden, whiplash-associated disorder (WAD) presents a major challenge. With annual North American incidence rates estimated to be between 70 and 329 per 100,000 people $(1,2)$, whiplash injuries are the most common injury following a motor vehicle collision (MVC) $(2,3)$. Although it is widely held that the majority of whiplash patients recover naturally within a few months after their injury, recent research (4) suggests that recovery is more prolonged and incomplete than previously believed, with an estimated $50 \%$ of patients still complaining of neck pain one year after injury. Moreover, WAD is associated with significant economic costs as a result of lost work productivity, medical care, legal services and other disability-related expenses $(5,6)$, with the majority of these expenses generated by patients with chronic symptoms (7). Given the scope and cost of WAD, developing effective therapies that prevent chronicity of pain and disability is of particular importance.

In 1995, the Quebec Task Force (QTF) published its benchmark review (1) of the scientific literature and expert opinion on WAD, redefining 'whiplash' as a construct and offering

${ }^{1}$ Lawson Health Research Institute; ${ }^{2}$ Department of Physical Medicine and Rehabilitation, Parkwood Hospital, St Joseph's Health Care; ${ }^{3}$ Schulich School of Medicine and Dentistry; ${ }^{4}$ School of Physical Therapy, Faculty of Health Sciences, University of Western Ontario, London, Ontario

Correspondence: Dr Robert W Teasell, Department of Physical Medicine and Rehabilitation, Parkwood Hospital, St Joseph's Health Care,

801 Commissioners Road East, London, Ontario N6C 5J1. Telephone 519-685-4000 ext 44559, fax 519-685-4023,

e-mail robert.teasell@sjh.london.on.ca 


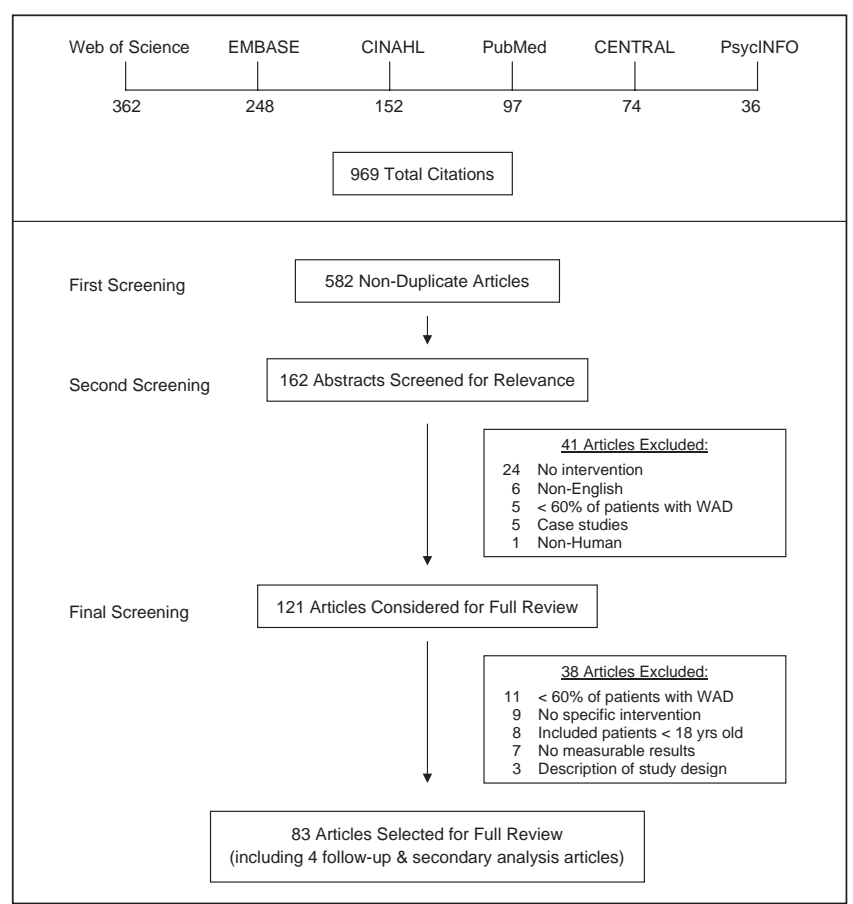

Figure 1) Article selection procedure. CENTRAL Cochrane Central Register of Controlled Trials; WAD Whiplash-associated disorder; yrs Years

guidelines for its management. According to the QTF, "whiplash is an acceleration deceleration mechanism of energy transfer to the neck, which...may result in bony or soft tissue injuries (whiplash injury), which in turn may lead to a variety of clinical manifestations (whiplash-associated disorders)" (1). One of the primary conclusions of this report was that the majority of therapeutic interventions used in the treatment of WAD had undergone little to no scientific investigation. Due to the paucity of scientifically rigorous studies, the QTF was forced to rely on consensus opinion for the majority of their mandated treatment recommendations, offering little in the way of evidence-based clinical guidelines. The QTF report emphasized the need for more and higher quality research.

More recently, Conlin et al $(8,9)$ conducted a systematic review of the whiplash treatment literature, which included studies published from 1993 to 2003. Conlin et al (8) found that the most effective intervention for WAD was simple exercise promoting mobilization of the neck initiated in the acute stage of injury. They noted further that, despite the QTF's recommendations, "remarkably little quality research" (9) had been published in the area of WAD management.

Since the review of Conlin et al $(8,9)$, many new studies have been published. The objective of the current review was to update and expand on previous work by evaluating the strength of evidence of therapies for acute (less than two weeks), subacute (two to 12 weeks) and chronic (longer than 12 weeks) WAD. Treatments were grouped according to time from injury to aid clinicians in deciding on an appropriate treatment course because therapies that are effective in the treatment of acute WAD may not necessarily be effective when initiated in the subacute or chronic phase. The present article, the first in a five-part series, provides an overview of the review methodology and a summary of the findings. Part 2 provides a review of acute WAD therapies while part 3 reviews interventions initiated during the subacute phase. Parts 4 and 5 provide reviews of noninvasive and invasive interventions for chronic WAD, respectively.

\section{Search strategy}

\section{METHOD}

A multistage screening process was conducted to identify all literature published from January 1980 to March 2009 that appeared to evaluate one or more therapeutic interventions for WAD. Multiple databases were searched (including PubMed, CINAHL, EMBASE, PsycINFO, Web of Science and the Cochrane Central Register of Controlled Trials [CENTRAL]) using the following search terms: whiplash AND (therapy OR treatment $\mathrm{OR}$ intervention $\mathrm{OR}$ rehabilitation $\mathrm{OR}$ surgery $\mathrm{OR}$ neurotomy). The literature search was limited to clinical studies written in English that examined adult (18 years of age and older) human populations. All nonduplicate titles were screened for relevance. The abstracts of any articles that appeared to evaluate a therapeutic intervention for WAD were retrieved for further screening. Full articles were retrieved and screened when the corresponding abstracts contained insufficient information to determine eligibility for the review. A graphic representation of the screening process is presented in Figure 1.

\section{Inclusion criteria}

Eligibility for inclusion was established a priori based on the following criteria:

- The purpose of the study was to examine the effect of a clearly defined treatment protocol for WAD (eg, provision of 'physiotherapy' was not considered to be a clearly defined protocol).

- At least $60 \%$ of participants in the study sample had experienced a whiplash injury resulting from an MVC, or the sample included a distinct and separately analyzed subgroup of whiplash patients.

- Evaluation of the treatment effect involved measurable outcomes.

- Sample was at least three participants with a whiplash injury.

Randomized controlled trials (RCTs), nonrandomized clinical trials and observational studies (such as cohort, case control and case series) were eligible for inclusion. Meta-analyses, reviews, abstracts, letters and case reports of single patients were read but excluded. The minimum sample size of three participants was chosen to exclude single case reports. No other methodological variables were reasons for exclusion.

\section{Methodological quality assessment and data abstraction} Information regarding the study sample size, source population, participant inclusion and exclusion criteria, follow-up duration and intervals, outcome measures and the results of statistical tests was abstracted using a standardized data abstraction form. Abstracted data were organized into tables; studies were grouped into the following eight categories according to treatment type:

1. Educational interventions: Interventions in which patients were instructed about the nature and course of WAD using evidence-based pamphlets, videos or personal communication.

2. Exercise programs: Programs that included various combinations of strength training, endurance training, 
stretching, aerobic exercises and functional exercises (eg, bending and lifting), typically targeting muscles in the neck.

3. Mobilization exercises: Exercises that were designed to promote mobility of the neck through small-range and small-amplitude neck exercises performed within the patient's comfort limit, or through advice to act as usual and maintain one's regular activities. These interventions were differentiated from exercise programs in that exercise programs have specific treatment aims (eg, strength training and endurance training), whereas mobilization programs are aimed at simply increasing or maintaining mobility.

4. Manual therapies: Interventions that used some form of passive manual manipulation or adjustment of the joints of the neck (eg, chiropractic adjustment).

5. Interdisciplinary interventions: Interventions that combined an exercise component with a psychological counselling component.

6. Pharmacological interventions: Interventions that examined the use of a pharmacological agent.

7. Surgical or injection-based interventions: Invasive interventions involving needles or a surgical procedure, more often intended for treating chronic WAD.

8. Alternative interventions: Interventions that did not fit into the previous intervention categories; these represented a wide variety of treatment approaches.

All of the RCTs that met inclusion criteria were evaluated for methodological quality using a standardized rating scale, the Physiotherapy Evidence Database (PEDro) scale. This evaluation tool was designed specifically for assessing physical therapy research and has been validated for the quality assessment of RCTs (10). The PEDro scale consists of 10 equally weighted yes/no questions relating to issues of methodological quality (Table 1) and can be accessed at www.pedro.org.au/ english/downloads/pedro-scale/. Two independent raters reviewed each article and discrepancies were resolved through consensus or, when that was not possible, by the review of a third rater. Studies with PEDro scores of 9 to 10 were considered to be of 'excellent' methodological quality, while scores of 6 to 8 were considered to be 'good' quality and scores of 4 to 5 were considered to be 'fair' quality. Studies scoring below 4 were judged to be of 'poor' quality and were considered to be methodologically equivalent to non-RCTs in terms of formulating conclusions. These descriptive terms of quality assessment were used to simplify the interpretation of results; however, it is important to note that these terms are only intended to provide an indication of a study's rating on the PEDro scale. Non-RCTs were not assigned a PEDro score and were instead given a 'no score' designation.

\section{Analysis}

Due to the relatively low number of studies investigating each of the specific WAD interventions, it was decided that both meta-analytical and levels-of-evidence approaches would be inappropriate. Therefore, a narrative approach was used to summarize the findings and formulate conclusions.

Because studies using a nonexperimental or uncontrolled design are generally considered to be of inferior quality, these types of studies were only used to formulate conclusions in the absence of RCTs or when the results of RCTs were conflicting.

\section{TABLE 1}

The Physiotherapy Evidence Database scale criteria

Subjects were randomly allocated to groups
Allocation was concealed
The groups were similar at baseline regarding the important prognostic
indicators
There was blinding of all subjects
There was blinding of all therapists who administered the therapy
There was blinding of all assessors who measured at least one key outcome
Measures of at least one key outcome were obtained from more than $85 \%$
of the subjects initially allocated to groups
All subjects for whom outcome data were available received the treatment
or control condition as allocated or, when this was not the case, data for at
least one key outcome were analyzed by intention to treat
The results of between-group statistical comparisons were reported for at
least one key outcome
The study provided both point estimates and measures of variability

Reproduced from reference 10

In addition, when the results of RCTs were conflicting, studies with higher PEDro scores were weighted more heavily. However, in some cases, it remained difficult to arrive at final conclusions using the above criteria (eg, when the results of a single study of higher quality conflicted with those of several studies of inferior quality). In these cases, we attempted to provide a rationale for our decision and to make the process as transparent as possible. In the end, the reader is encouraged to be a 'critical consumer' of all of the material presented.

\section{RESULTS}

In total, 83 studies (40 RCTs, four follow-up studies and 39 nonRCTs) were identified that evaluated therapies across the continuum of WAD and met our inclusion criteria. The median PEDro score of the RCTs was 6, with scores ranging from 2 to 9 (Table 2). The most common methodological limitations of the 40 RCTs were failing to blind patients (30\%) and therapists (25\%), and not conducting analyses on an intention-to-treat basis $(28 \%)$, with less than one-third of studies using these methods. Regarding the distribution of research across the WAD continuum, there were a greater number of studies investigating chronic WAD compared with either acute or subacute WAD (47 versus 23 and 13, respectively). However, while the RCTs evaluating chronic WAD were generally of better methodological quality, a greater proportion of the studies investigating chronic WAD were non-RCTs.

\section{Interventions for acute WAD}

In total, 23 studies were identified that evaluated an intervention initiated during the acute stage of WAD (ie, within two weeks of injury), 16 of which were RCTs. For the treatment of acute WAD, there is strong evidence to suggest that immobilization with a soft collar is not only ineffective but may actually impede recovery. Conversely, although exercise programs, active mobilization and advice to act as usual all appear to improve recovery, it is not clear which of these interventions is the most effective. In addition to activation-based treatments, there is also some evidence suggesting that both pulsed electromagnetic field therapy and methylprednisolone infusion may improve recovery over the short term; however, the literature supporting both of these interventions is very limited. In contrast, it has not been demonstrated that laser acupuncture 
TABLE 2

Physiotherapy Evidence Database (PEDro) quality assessment scores for all included randomized controlled trials

\begin{tabular}{|c|c|c|c|c|c|c|c|c|c|c|c|}
\hline \multirow[b]{2}{*}{ Reference, year } & \multicolumn{10}{|c|}{ PEDro criteria } & \multirow[b]{2}{*}{ Total score } \\
\hline & RA & CA & BS & SB & TB & $A B$ & AF & ITT & $\mathrm{BC}$ & PVM & \\
\hline \multicolumn{12}{|l|}{ Acute } \\
\hline Ferrari et al (19), 2005 & $\checkmark$ & $\checkmark$ & $\checkmark$ & & $\checkmark$ & $\checkmark$ & $\checkmark$ & & $\checkmark$ & $\checkmark$ & 8 \\
\hline Pettersson and Toolanen (20), 1998 & $\checkmark$ & $\checkmark$ & $\checkmark$ & $\checkmark$ & $\checkmark$ & $\checkmark$ & $\checkmark$ & & $\checkmark$ & & 8 \\
\hline Foley-Nolan et al (21), 1992 & $\checkmark$ & $\checkmark$ & $\checkmark$ & $\checkmark$ & $\checkmark$ & $\checkmark$ & $\checkmark$ & & $\checkmark$ & & 8 \\
\hline Vassiliou et al (22), 2006 & $\checkmark$ & $\checkmark$ & $\checkmark$ & & & & $\checkmark$ & $\checkmark$ & $\checkmark$ & $\checkmark$ & 7 \\
\hline Kongsted et al (23), 2008 & $\checkmark$ & $\checkmark$ & & & & & $\checkmark$ & $\checkmark$ & $\checkmark$ & $\checkmark$ & 6 \\
\hline McKinney et al (24), 1989 & $\checkmark$ & $\checkmark$ & $\checkmark$ & & & $\checkmark$ & & & $\checkmark$ & $\checkmark$ & 6 \\
\hline Kongsted et al (25), 2007 & $\checkmark$ & $\checkmark$ & & & & & $\checkmark$ & $\checkmark$ & $\checkmark$ & $\checkmark$ & 6 \\
\hline Mealy et al (14), 1986 & $\checkmark$ & $\checkmark$ & $\checkmark$ & & & $\checkmark$ & & & $\checkmark$ & $\checkmark$ & 6 \\
\hline Dehner et al (26), 2006 & $\checkmark$ & & $\checkmark$ & & & & $\checkmark$ & & $\checkmark$ & $\checkmark$ & 5 \\
\hline Schnabel et al (11), 2004 & $\checkmark$ & $\checkmark$ & $\checkmark$ & & & & & & $\checkmark$ & $\checkmark$ & 5 \\
\hline Aigner et al (27), 2006 & $\checkmark$ & & & $\checkmark$ & & & $\checkmark$ & & $\checkmark$ & $\checkmark$ & 5 \\
\hline Borchgrevink et al (28), 1998 & $\checkmark$ & & $\checkmark$ & & & $\checkmark$ & & & $\checkmark$ & $\checkmark$ & 5 \\
\hline Rosenfeld et al (12), 2000 & $\checkmark$ & & $\checkmark$ & & & & $\checkmark$ & & $\checkmark$ & & 4 \\
\hline Dehner et al (29), 2009 & $\checkmark$ & $\checkmark$ & & & & & & $\checkmark$ & $\checkmark$ & & 4 \\
\hline Soderlund (30), 2000 & $\checkmark$ & & $\checkmark$ & & & & & & $\checkmark$ & $\checkmark$ & 4 \\
\hline Thuile and Walzl (31), 2002 & $\checkmark$ & & $\checkmark$ & & & & & & $\checkmark$ & $\checkmark$ & 4 \\
\hline \multicolumn{12}{|l|}{ Subacute } \\
\hline Bunketorp et al (32), 2006 & $\checkmark$ & $\checkmark$ & $\checkmark$ & & & $\checkmark$ & $\checkmark$ & $\checkmark$ & $\checkmark$ & $\checkmark$ & 8 \\
\hline Carroll (33), 2008 & $\checkmark$ & $\checkmark$ & & $\checkmark$ & $\checkmark$ & $\checkmark$ & & & $\checkmark$ & $\checkmark$ & 7 \\
\hline Scholten-Peeters et al (15), 2006 & $\checkmark$ & $\checkmark$ & & & & $\checkmark$ & $\checkmark$ & $\checkmark$ & $\checkmark$ & $\checkmark$ & 7 \\
\hline Fernández-des-las-Peñas et al (34), 2004 & $\checkmark$ & & $\checkmark$ & & & & $\checkmark$ & & $\checkmark$ & $\checkmark$ & 5 \\
\hline Provinciali et al (35), 1996 & $\checkmark$ & & $\checkmark$ & & & $\checkmark$ & $\checkmark$ & & $\checkmark$ & & 5 \\
\hline Fernández-des-las-Peñas et al (36), 2004 & $\checkmark$ & & $\checkmark$ & & & & & & $\checkmark$ & $\checkmark$ & 4 \\
\hline \multicolumn{12}{|l|}{ Chronic (noninvasive treatment) } \\
\hline Jull et al (37), 2007 & $\checkmark$ & $\checkmark$ & $\checkmark$ & & & $\checkmark$ & $\checkmark$ & $\checkmark$ & $\checkmark$ & $\checkmark$ & 8 \\
\hline Stewart et al (38), 2007 & $\checkmark$ & $\checkmark$ & $\checkmark$ & & & $\checkmark$ & $\checkmark$ & $\checkmark$ & $\checkmark$ & $\checkmark$ & 8 \\
\hline Ryan (39), 2006 & $\checkmark$ & & $\checkmark$ & $\checkmark$ & & $\checkmark$ & $\checkmark$ & $\checkmark$ & $\checkmark$ & $\checkmark$ & 8 \\
\hline Wicksell et al (40), 2008 & $\checkmark$ & $\checkmark$ & $\checkmark$ & & & & $\checkmark$ & $\checkmark$ & $\checkmark$ & $\checkmark$ & 7 \\
\hline Vikne et al (41), 2007 & $\checkmark$ & $\checkmark$ & $\checkmark$ & & & $\checkmark$ & & & $\checkmark$ & $\checkmark$ & 6 \\
\hline Soderlund and Lindberg (42), 2001 & $\checkmark$ & & $\checkmark$ & & & $\checkmark$ & $\checkmark$ & & $\checkmark$ & $\checkmark$ & 6 \\
\hline van Wieringen et al (43), 2001 & $\checkmark$ & & & $\checkmark$ & $\checkmark$ & $\checkmark$ & & & $\checkmark$ & $\checkmark$ & 6 \\
\hline Klobas et al (44), 2006 & $\checkmark$ & & $\checkmark$ & & & $\checkmark$ & $\checkmark$ & & $\checkmark$ & & 5 \\
\hline Hansson et al (45), 2006 & $\checkmark$ & & & & & $\checkmark$ & & $\checkmark$ & $\checkmark$ & & 4 \\
\hline Humphreys and Irgens (46), 2002 & $\checkmark$ & & & & & & $\checkmark$ & & $\checkmark$ & $\checkmark$ & 4 \\
\hline Ventegodt et al (47), 2004 & $\checkmark$ & & $\checkmark$ & & & & & & $\checkmark$ & $\checkmark$ & 4 \\
\hline Fitz-Ritson (48), 1995 & $\checkmark$ & & & & & & $\checkmark$ & & & & 2 \\
\hline \multicolumn{12}{|c|}{ Chronic (surgical or injection-based treatment) } \\
\hline Braker et al (17), 2008 & $\checkmark$ & $\checkmark$ & $\checkmark$ & $\checkmark$ & $\checkmark$ & $\checkmark$ & $\checkmark$ & & $\checkmark$ & $\checkmark$ & 9 \\
\hline Padberg et al (49), 2007 & $\checkmark$ & $\checkmark$ & $\checkmark$ & $\checkmark$ & $\checkmark$ & $\checkmark$ & $\checkmark$ & & $\checkmark$ & $\checkmark$ & 9 \\
\hline Lord et al (50), 1996 & $\checkmark$ & $\checkmark$ & $\checkmark$ & $\checkmark$ & $\checkmark$ & $\checkmark$ & $\checkmark$ & & $\checkmark$ & & 8 \\
\hline Freund and Schwartz (51), 2000 & $\checkmark$ & & $\checkmark$ & $\checkmark$ & $\checkmark$ & & $\checkmark$ & & $\checkmark$ & $\checkmark$ & 7 \\
\hline Barnsley et al (52), 1994 & $\checkmark$ & & $\checkmark$ & $\checkmark$ & $\checkmark$ & $\checkmark$ & $\checkmark$ & & $\checkmark$ & & 7 \\
\hline Byrn et al (53), 1993 & $\checkmark$ & $\checkmark$ & $\checkmark$ & $\checkmark$ & & & $\checkmark$ & & $\checkmark$ & & 6 \\
\hline
\end{tabular}

AB Assessor blinding; AF Adequate follow-up; BC Between-group comparisons; BS Baseline similarity; CA Concealed allocation; ITT Intention-to-treat analysis; PVM Point estimates and variability reported; RA Random allocation; SB Subject blinding; TB Therapist blinding

or educational interventions are effective in improving recovery from acute WAD.

\section{Interventions for subacute WAD}

In total, 13 articles were identified that evaluated an intervention initiated during the subacute stage of WAD (ie, between two and 12 weeks following injury), six of which were RCTs. For the treatment of subacute WAD, the use of both exercise programs and botulinum toxin A (BTXA) injections were not supported by the literature. In contrast, there is some, albeit weak, evidence that both interdisciplinary interventions and manual joint manipulation may provide some benefit during the subacute phase of WAD; however, given that the evidence for both of these interventions was derived from studies that were completely unblinded and only followed patients for a short period of time, further research is needed before firm conclusions can be drawn regarding the effectiveness of these two treatments. 


\section{Noninvasive interventions for chronic WAD}

In total, 22 articles were identified that evaluated noninvasive interventions initiated during the chronic stage of WAD (ie, more than 12 weeks after injury), 12 of which were RCTs. For the treatment of chronic WAD, exercise programs appeared to be the most effective noninvasive intervention for chronic WAD, although many questions remain regarding the relative effectiveness of different exercise protocols. While the majority of studies support the effectiveness of interdisciplinary interventions, the results of the only two RCTs provide conflicting results; nevertheless, there have been some promising findings, particularly regarding the use of acceptance-based cognitive behavioural therapy to reduce whiplash-related disability. There is also some evidence in favour of manual joint manipulation and myofeedback training. However, these treatments are only supported by one small non-RCT each and, as such, the evidence is insufficient to establish the effectiveness of either of these treatments. Finally, there is a lack of evidence supporting the use of either melatonin supplements or 'alternative' therapies in improving whiplash-related symptoms.

Surgical and injection-based interventions for chronic WAD In total, 25 articles were identified that evaluated surgical and/or injection-based interventions initiated during the chronic stage of WAD (ie, more than 12 weeks after injury), six of which were RCTs. For the treatment of chronic WAD, there is moderate evidence that radiofrequency neurotomy is an effective treatment for whiplash-related pain, although relief is not permanent. Sterile water injections have also been demonstrated to be superior to saline injections; nevertheless, further research is needed to determine whether this treatment is actually beneficial. Although there is limited evidence supporting a wide range of other interventions (eg, carpal tunnel decompression), each of these was evaluated by a single nonRCT only and, as such, the evidence was insufficient to support the use of any of these treatments. There is conflicting evidence regarding the effectiveness of BTXA injections. While only one of three RCTs found that BTXA injections were associated with significant benefit compared with placebo, one of the other trials reported a consistent trend in favour of BTXA over placebo.

\section{DISCUSSION}

As a common consequence of MVCs, WAD is a cause of considerable suffering and disability, and a significant amount of resources are invested into its treatment. Despite this, our review discovered a relatively small number of studies $(n=83)$ examining therapeutic interventions for WAD and an even smaller number of RCTs $(n=40)$. Moreover, only $60 \%$ of the RCTs were rated as good or excellent in terms of methodological quality (ie, PEDro score of 6 or more). In many cases, our ability to formulate conclusions with confidence was limited by inadequate methodological rigour.

Overall, exercise and mobilization programs were the most frequently investigated interventions (20 RCTs), and this was the only type of therapy evaluated during all three stages of recovery (ie, acute, subacute and chronic). Considering all available evidence, exercise and/or mobilization-based therapies currently have the greatest level of evidence in support of their effectiveness for reducing the duration and severity of whiplash-associated neck pain, range of motion deficits and disability, at least during the acute and chronic stages of the disorder. While the literature does not support the use of activation-based therapies during the subacute stage, this may be due more to a lack of research than to a lack of effectiveness. Although it seems likely that exercise programs are beneficial for patients across the WAD continuum, further research is needed to establish the effectiveness of activation-based therapies for patients with subacute WAD.

There is also little information regarding the relative effectiveness of various exercise regimens. For example, it is not clear which type of activation-based therapy (ie, exercise, simple mobilization or advice to remain active) is most effective for patients with acute WAD. There is, however, a substantial amount of evidence suggesting that immobilization of the neck using a soft cervical collar is not only ineffective, but that it may even impede natural recovery $(11-14)$. Conversely, there is moderate evidence that aggressive strengthening programs $(15,16)$ may have adverse long-term effects on pain and functional recovery. Thus, while it appears that it is important to keep whiplash patients active and mobilized, aggressive workhardening programs should be avoided, at least during the first three months after injury. Although exercise programs are helpful, aggressive exercise programs may not be.

Interdisciplinary interventions were the second most frequently studied type of treatment, although only three RCTs were identified (one initiated during the subacute stage, and two during the chronic stage). The results from two of the RCTs suggest that the addition of psychological counselling to a course of physiotherapy is significantly more effective than physiotherapy alone, while the other RCT found no difference between patients who received counselling and those who did not. It is important to note, however, that the trial that reported no significant group differences provided the clearest comparison of physiotherapy plus counselling to physiotherapy alone. Unfortunately, patients were not blinded in any of these studies, severely limiting the strength of conclusions that can be drawn. Considering all of the available evidence, from both randomized and nonrandomized trials, the overwhelming majority of studies supported the use of interdisciplinary interventions; however, formulating conclusions based on this evidence is complicated by the heterogeneity of the interventions and the methodological shortcomings of the research. Thus, further research is needed before definitive conclusions can be drawn regarding the effectiveness of interdisciplinary interventions.

The use of BTXA injections was evaluated during both the subacute and chronic stages of WAD. While only one of four RCTs found that BTXA injections were associated with significant benefit compared with placebo, two of the other trials reported a trend in favour of BTXA over placebo. Furthermore, there is some indication that these disparate findings may be related to the actual dosages and timing of the treatment (17). Although further research is needed to explore these differences in BTXA administration, BTXA injections represent a promising but, as yet, unproven intervention in the treatment of WAD.

The present review was limited by several methodological concerns. First, because of the small number of studies in the whiplash literature, the criteria for inclusion were quite broad. 
TABLE 3

Summary of evidence for noninvasive whiplash-associated disorder (WAD) therapies

\begin{tabular}{|c|c|}
\hline Intervention & Conclusion \\
\hline Educational & It does not appear that providing educational information during the acute phase provides significant benefit \\
\hline Exercise programs & $\begin{array}{l}\text { It appears that exercise programs are effective in reducing short-term pain intensity in both the acute and chronic stages of WAD, although it } \\
\text { does not appear that these programs influence long-term recovery. In contrast, there is some evidence that aggressive strengthening } \\
\text { programs may be counterproductive, particularly during the subacute stage of WAD }\end{array}$ \\
\hline Mobilization & $\begin{array}{l}\text { There is strong evidence that immobilization with a soft collar during the acute phase of WAD is less effective than active mobilization and no } \\
\text { more effective than advice to act as usual. In contrast, active mobilization during the acute phase of WAD appears to be associated with } \\
\text { reduced pain intensity, although it is not clear whether active mobilization is any more effective than advice to act as usual }\end{array}$ \\
\hline Interdisciplinary & $\begin{array}{l}\text { Although the majority of studies support its benefit, there is conflicting evidence regarding the effectiveness of interdisciplinary interventions } \\
\text { for patients with either subacute or chronic WAD. Further research is needed, particularly regarding the use of acceptance-based cognitive } \\
\text { behavioural therapy to reduce whiplash-related disability }\end{array}$ \\
\hline $\begin{array}{l}\text { Chiropractic } \\
\text { manipulation }\end{array}$ & $\begin{array}{l}\text { Although there is some evidence that chiropractic manipulation may be of short-term benefit to patients with either subacute or chronic WAD, } \\
\text { the evidence is insufficient to determine the effectiveness of this treatment }\end{array}$ \\
\hline Pharmacological & $\begin{array}{l}\text { There is some evidence that methylprednisolone infusion is effective in improving recovery from acute WAD; however, further research using } \\
\text { larger sample sizes is needed before any conclusions can be drawn regarding its effectiveness. In contrast, it does not appear that } \\
\text { melatonin supplements are effective in reducing chronic whiplash-related symptoms }\end{array}$ \\
\hline Injection based & $\begin{array}{l}\text { There is contradictory evidence regarding the effectiveness of botulinum toxin injections during the chronic stage of WAD, and no evidence } \\
\text { that they are beneficial during the subacute stage. There is also no evidence supporting the use of intra-articular or selective nerve root } \\
\text { block corticosteroid injections in the treatment of chronic WAD. While there is limited evidence supporting a number of other injection-based } \\
\text { interventions, the evidence is insufficient to establish the effectiveness of any of these treatments }\end{array}$ \\
\hline Surgical & $\begin{array}{l}\text { There is moderate evidence that radiofrequency neurotomy is effective in reducing pain in patients with chronic WAD, although relief is not } \\
\text { permanent. While there is also some evidence suggesting that occipital nerve decompression, carpal tunnel decompression, and cervical } \\
\text { discectomy and fusion provide some benefit to patients with chronic WAD, the evidence is insufficient to establish the effectiveness of any } \\
\text { of these treatments }\end{array}$ \\
\hline Alternative & $\begin{array}{l}\text { Although there is some evidence that pulsed electromagnetic field therapy decreases pain intensity and increases cervical range of motion } \\
\text { over the short term for patients with acute WAD, the evidence is insufficient to support the use of this treatment. Moreover, laser } \\
\text { acupuncture does not appear to be any more effective than placebo in the treatment of acute WAD. Based on the results of one small case } \\
\text { study, there is limited evidence that myofeedback training may be beneficial for some patients with chronic WAD; however, further research } \\
\text { is needed to determine which patients are most likely to benefit from this intervention. The use of alternative therapies does not appear to } \\
\text { be beneficial for patients with chronic WAD }\end{array}$ \\
\hline
\end{tabular}

All studies were included regardless of study design, as long as $60 \%$ of the sample experienced WAD and they included a sample of at least three participants. This may have resulted in the inclusion of some studies of lower scientific merit; however, such studies were used to formulate conclusions only in the absence of superior RCTs. Second, there are limitations with the assessment process used in the current review to evaluate the methodological quality of RCTs. For example, it is possible that an RCT with significant between-group differences at baseline that did not blind patients, therapists or assessors could still have received a PEDro score of 6 and be considered a study of good methodological quality despite these significant limitations. Again, these issues were noted in relevant conclusions and study descriptions.

Related to the issue of PEDro scoring, it should be noted that it is quite difficult to effectively blind patients and therapists in many types of therapy studies simply because of the nature of the interventions (18). Thus, for many of the studies included in the present review, the maximum PEDro score they could be expected to attain would be 8 . Specifically, of the 33 RCTs investigating noninvasive interventions, patients and therapists were blinded in only $15 \%$ and $12 \%$ of studies, respectively. The inability to conduct studies in a blinded fashion is a major limitation of the WAD literature because this has the potential to introduce significant bias. This shortcoming can be mitigated to some extent by the inclusion of blinded assessors, which was the case in slightly more than one-half of the included studies.

A limitation of the WAD literature is that patients are rarely classified on the basis of injury severity. The QTF proposed a clinical classification system ranging from 0 (no complaint or physical injury) to 4 (neck complaint and fracture or dislocation) (1). The vast majority of whiplash injuries presented in these studies as well as in clinical practice would likely be classified as grade 2, at least in studies examining patients during the acute and subacute stages of recovery. Severity of injury would presumably have an impact on treatment outcomes because patients with less severe injuries should, as a group, take less time to recover and experience greater spontaneous recovery.

The therapies that have been advocated for patients with WAD are quite diverse, which likely reflects several factors including the variable nature of WAD, our incomplete understanding of the disorder and the absence of any one treatment distinguishing itself as the most effective. These issues are further complicated by adversarial legal systems and restrictive clinical guidelines that arise in the absence of good research evidence. Based on the available evidence, exercise and mobilization programs have the strongest supporting evidence for treatment of acute and chronic WAD, although many questions remain regarding the relative effectiveness of various protocols. Interestingly, there is insufficient evidence to support any treatment for subacute WAD. For patients with chronic WAD who do not respond to conventional treatments, it appears that radiofrequency neurotomy may be the most effective treatment option. While several conclusions were reached in the present review (Table 3), there is a lack of research investigating the efficacy of interventions for patients at any stage on the WAD continuum. Clearly, further research is needed to determine which treatments are most effective at reducing the disabling symptoms associated with WAD. 


\section{REFERENCES}

1. Spitzer WO, Skovron ML, Salmi LR, et al. Scientific monograph of the Quebec Task Force on whiplash-associated disorders: Redefining 'whiplash' and its management. Spine 1995;20:2S-73S.

2. Quinlan KP, Annest JL, Myers B, Ryan G, Hill H. Neck strains and sprains among motor vehicle occupants - United States, 2000. Accid Anal Prev 2004;36:21-7.

3. Berglund A, Alfredsson L, Jensen I, Bodin L, Nygren A. Occupant-and crash-related factors associated with risk of whiplash injury. Ann Epidemiol 2003;13:66-72.

4. Carroll LJ, Holm LW, Hogg-Johnson S, et al. Course and prognostic factors for neck pain in whiplash-associated disorders (WAD): Results of the bone and joint decade 2000-2010 task force on neck pain and its associated disorders. Spine 2008;33(4 Suppl):S83-92.

5. Yoganandan N, Pintar FA, Kleinberger M. Whiplash injury. Biomechanical experimentation. Spine 1999;24:83-5.

6. Freeman MD, Croft AC, Rossignol AM, Weaver DS, Reiser M. A review and methodologic critique of the literature refuting whiplash syndrome. Spine 1999;24:86-98.

7. Galasko SB. The cost of whiplash-associated disorders. In: Gunzburg R, Szpalski M, eds. Whiplash Injuries: Current Concepts in the Prevention, Diagnosis and Treatment of the Cervical Whiplash Syndrome. Philidelphia: Lippincott-Raven, 1998:283-90.

8. Conlin A, Bhogal S, Sequeira K, Teasell R. Treatment of whiplash-associated disorders - part I: Noninvasive interventions. Pain Res Manage 2005;10:21-32.

9. Conlin A, Bhogal S, Sequeira K, Teasell R. Treatment of whiplash-associated disorders - part II: Medical and surgical interventions. Pain Res Manage 2005;10:33-40.

10. Maher CG, Sherrington C, Hebert RD, Mosely AM, Elkins M. Reliability of the PEDro scale for rating quality of randomized controlled trials. Phys Ther 2003;83:713-21.

11. Schnabel M, Ferrari R, Vassiliou T, Kaluza G. Randomised, controlled outcome study of active mobilisation compared with collar therapy for whiplash injury. Emerg Med J 2004;21:306-10.

12. Rosenfeld M, Gunnarsson R, Borenstein P. Early intervention in whiplash-associated disorders: A comparison of two treatment protocols. Spine 2000;25:1782-7.

13. Rosenfeld M, Seferiadis A, Carlsson J, Gunnarsson R. Active intervention in patients with whiplash-associated disorders improves long-term prognosis: A randomized controlled clinical trial. Spine 2003;28:2491-8.

14. Mealy K, Brennan H, Fenelon GC. Early mobilization of acute whiplash injuries. Br Med J (Clin Res Ed) 1986;292:656-7.

15. Scholten-Peeters GG, Neeleman-van der Steen CW, van der Windt DA, Hendriks EJ, Verhagen AP, Oostendorp RA. Education by general practitioners or education and exercises by physiotherapists for patients with whiplash-associated disorders? A randomized clinical trial. Spine 2006;31:723-31.

16. Cassidy JD, Carroll LJ, Cote P, Frank J. Does multidisciplinary rehabilitation benefit whiplash recovery? Results of a population-based incidence cohort study. Spine 2007;32:126-31.

17. Braker C, Yariv S, Adler R, Badarny S, Eisenberg E. The analgesic effect of botulinum-toxin A on postwhiplash neck pain. Clin J Pain 2008;24:5-10.

18. Bhogal SK, Teasell RW, Foley NC, Speechley MR. The PEDro scale provides a more comprehensive measure of methodological quality than the Jadad scale in stroke rehabilitation literature. J Clin Epidemiol 2005;58:668-73.

19. Ferrari R, Rowe BH, Majumdar SR, et al. Simple educational intervention to improve the recovery from acute whiplash: Results of a randomized, controlled trial. Acad Emerg Med 2005;12:699-706.

20. Pettersson K, Toolanen G. High-dose methylprednisolone prevents extensive sick leave after whiplash injury: A prospective, randomized, double-blind study. Spine 1998;23:984-9.

21. Foley-Nolan D, Moore K, Codd M, Barry C, O'Connor P, Coughlan RJ. Low energy high frequency pulsed electromagnetic therapy for acute whiplash injuries. A double blind randomized controlled study. Scand J Rehabil Med 1992;24:51-9.

22. Vassiliou T, Kaluza G, Putzke C, Wulf H, Schnabel M. Physical therapy and active exercises - an adequate treatment for prevention of late whiplash syndrome? Randomized controlled trial in 200 patients. Pain 2006;124:69-76.
23. Kongsted A, Qerama E, Kasch H, et al. Education of patients after whiplash injury: Is oral advice any better than a pamphlet? Spine 2008;33:E843-48.

24. McKinney LA, Dornan JO, Ryan M. The role of physiotherapy in the management of acute neck sprains following road-traffic accidents. Arch Emerg Med 1989;6:27-33.

25. Kongsted A, Qerama E, Kasch H, et al. Neck collar, 'act-as-usual' or active mobilization for whiplash injury? A randomized parallel-group trial. Spine 2007;32:618-26.

26. Dehner C, Hartwig E, Strobel P, et al. Comparison of the relative benefits of 2 versus 10 days of soft collar cervical immobilization after acute whiplash injury. Arch Phys Med Rehabil 2006;87:1423-7.

27. Aigner N, Fialka C, Radda C. Adjuvant laser acupuncture in the treatment of whiplash injuries: A prospective randomized placebo-controlled trial. Wien Klin Wochenschr 2006;118:95-9.

28. Borchgrevink GE, Kaasa A, McDonagh D, Stiles TC, Haraldseth O, Lereim I. Acute treatment of whiplash neck sprain injuries. A randomized trial of treatment during the first 14 days after a car accident. Spine 1998;23:25-31.

29. Dehner C, Elbel M, Strobel P, et al. Grade II whiplash injuries to the neck: What is the benefit for patients treated by different physical therapy modalities? Patient Saf Surg 2009;3:2.

30. Soderlund A. Acute whiplash-associated disorders (WAD): The effects of early mobilization and prognostic factors in long-term symptomology. Clin Rehabil 2000;14:457-67.

31. Thuile C, Walzl M. Evaluation of electromagnetic fields in the treatment of pain in patients with lumbar radiculopathy or the whiplash syndrome. NeuroRehabilitation 2002;17:63-7.

32. Bunketorp L, Lindh M, Carlsson J, Stener-Victorin E. The effectiveness of a supervised physical training model tailored to the individual needs of patients with whiplash-associated disorders a randomized controlled trial. Clin Rehabil 2006;20:201-17.

33. Carroll A. A prospective randomized controlled study of the role of botulinum toxin in whiplash-associated disorder. Clin Rehabil 2008;22:513-9.

34. Fernández-des-las-Peñas C, Fernandez-Carnero J, Fernandez AP, Lomas-Vega R, Miangolarra-Page JC. Dorsal manipulation in whiplash injury treatment: A randomized controlled trial. J Whiplash Relat Disord 2004;3:55-72.

35. Provinciali L, Baroni M, Illuminati L, Ceravolo MG. Multimodal treatment to prevent the late whiplash syndrome. Scand J Rehabil Med 1996;28:105-11.

36. Fernández-des-las-Peñas C, Fernandez-Carnero J, Palomeque del Cerro L, Miangolarra-Page JC. Manipulative treatment vs. conventional physiotherapy treatment in whiplash injury: A randomized controlled trial. J Whiplash Relat Disord 2004;3:73-90.

37. Jull G, Sterling M, Kenardy J, Beller E. Does the presence of sensory hypersensitivity influence outcomes of physical rehabilitation for chronic whiplash? A preliminary RCT. Pain 2007;129:28-34.

38. Stewart MJ, Maher CG, Refshauge KM, Herbert RD, Bogduk N, Nicholas M. Randomized controlled trial of exercise for chronic whiplash-associated disorders. Pain 2007;128:59-68.

39. Ryan JM. Reducing pain and disability for whiplash victims: A double-blind randomised controlled trial. Cochrane Central Register of Controlled Trials 2006;(3):CN00477965.

40. Wicksell RK, Ahlqvist J, Bring A, Melin L, Olsson GL. Can exposure and acceptance strategies improve functioning and life satisfaction in people with chronic pain and whiplash associated disorders (WAD)? A randomized controlled trial. Cogn Behav Ther 2008;37:169-82.

41. Vikne J, Oedegaard A, Laerum E, Ihlebaek C, Kirkesola G. A randomized study of new sling exercise treatment vs. traditional physiotherapy for patients with chronic whiplash-associated disorders with unsettled compensation claims. J Rehabil Med 2007;39:252-9.

42. Soderlund A, Lindberg P. Cognitive behavioural components in physiotherapy management of chronic whiplash associated disorders (WAD) - a randomised group study. Physiother Theory Pract 2001;17:229-38.

43. van Wieringen S, Jansen T, Smits MG. Melatonin for chronic whiplash syndrome with delayed melatonin onset - 
randomised, placebo-controlled trial. Clin Drug Investig 2001;21:813-20.

44. Klobas L, Axelsson S, Tegelberg A. Effect of therapeutic jaw exercise on temporomandibular disorders in individuals with chronic whiplash-associated disorders. Acta Odontologica Scandinavica 2006;64:341-7.

45. Hansson EE, Mansson N, Ringsberg KAM, Hakansson A. Dizziness among patients with whiplash-associated disorder: A randomized controlled trial. J Rehabil Med 2006;38:387-90.

46. Humphreys BK, Irgens PM. The effect of a rehabilitation exercise program on head repositioning accuracy and reported levels of pain in chronic neck pain subjects. J Whiplash Relat Disord 2002;1:99-112.

47. Ventegodt S, Merrick J, Andersen NJ, Bendix T. A combination of Gestalt therapy, Rosen body work, and craniosacral therapy did not help in chronic whiplash-associated disorders (WAD) results of a randomized clinical trial. ScientificWorldJournal 2004;4:1055-68.
48. Fitz-Ritson D. Phasic exercises for cervical rehabilitation after 'whiplash' trauma. J Manipulative Physiol Ther 1995;18:21-4.

49. Padberg M, de Bruijn SFTM, Tavy DLJ. Neck pain in chronic whiplash syndrome treated with botulinum toxin. A double blind, placebo-controlled clinical trial. J Neurol 2007;254:290-5.

50. Lord SM, Barnsley L, Wallis BJ, McDonald GJ, Bogduk N. Percutaneous radio-frequency neurotomy for chronic cervical zygapophyseal-joint pain. N Engl J Med 1996;335:1721-6.

51. Freund BJ, Schwartz M. Treatment of whiplash associated neck pain [corrected] with botulinum toxin-A: A pilot study. J Rheumatol 2000;27:481-4.

52. Barnsley L, Lord SM, Wallis BJ, Bogduk N. Lack of effect of intraarticular corticosteroids for chronic pain in the cervical zygapophyseal joints. N Engl J Med 1994;330:1047-50.

53. Byrn C, Olsson I, Falkheden L, et al. Subcutaneous sterile water injections for chronic neck and shoulder pain following whiplash injuries. Lancet 1993;341:449-52. 


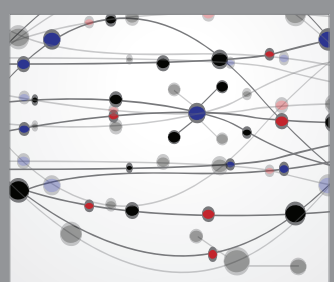

The Scientific World Journal
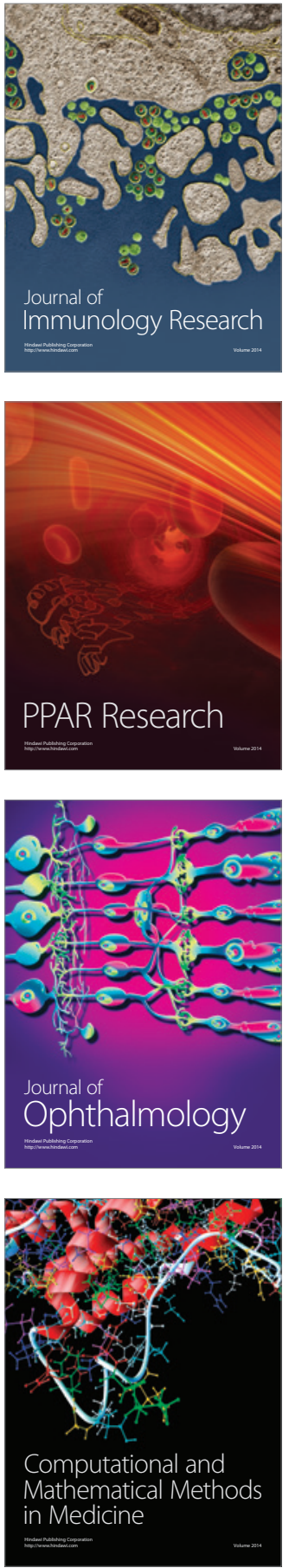

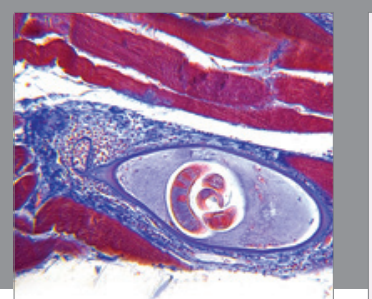

Gastroenterology Research and Practice

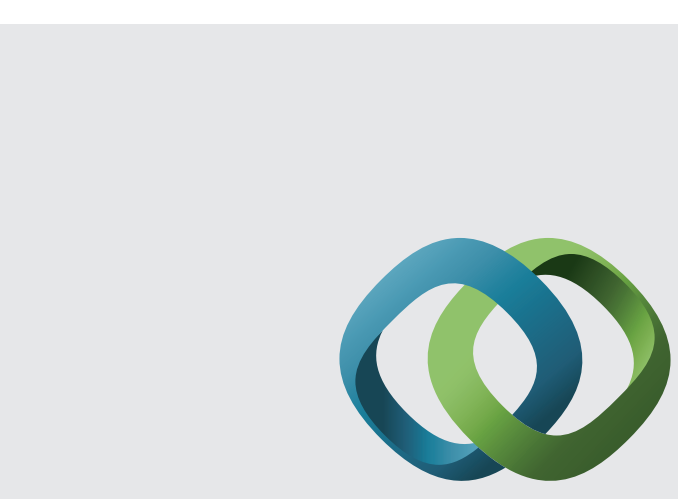

\section{Hindawi}

Submit your manuscripts at

http://www.hindawi.com
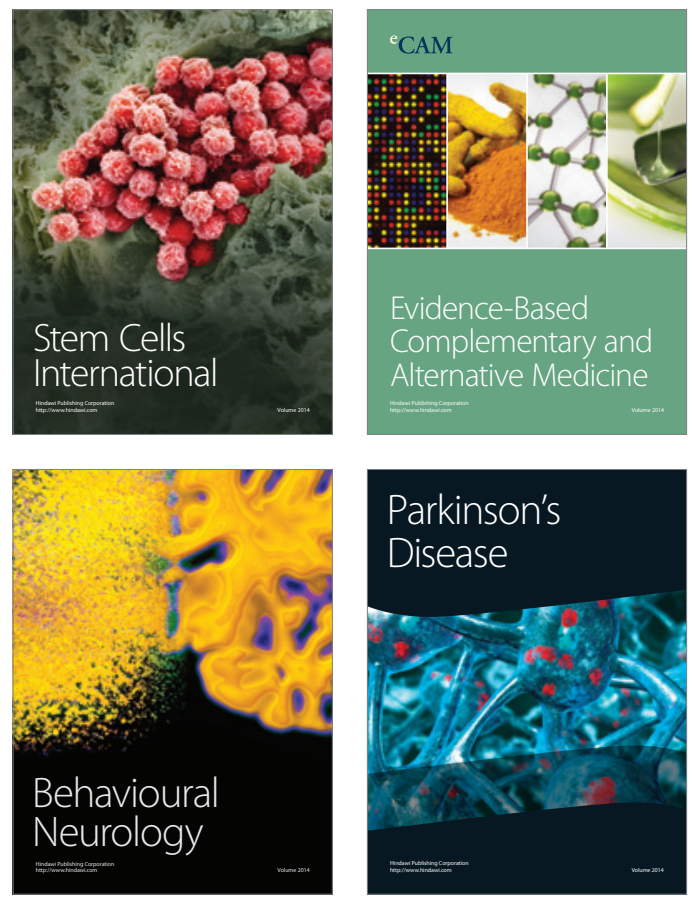
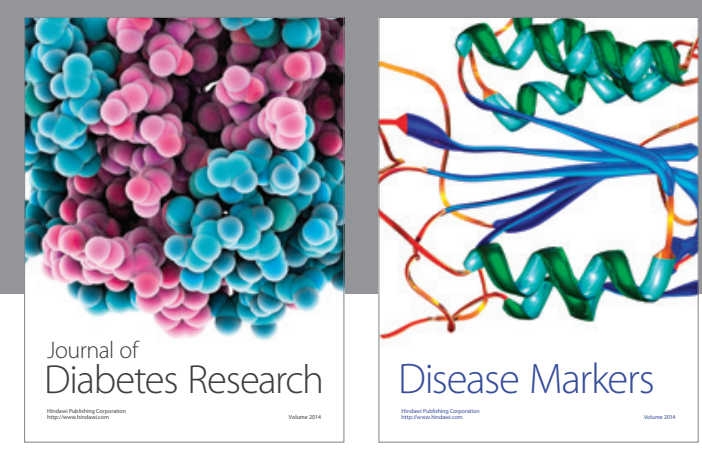

Disease Markers
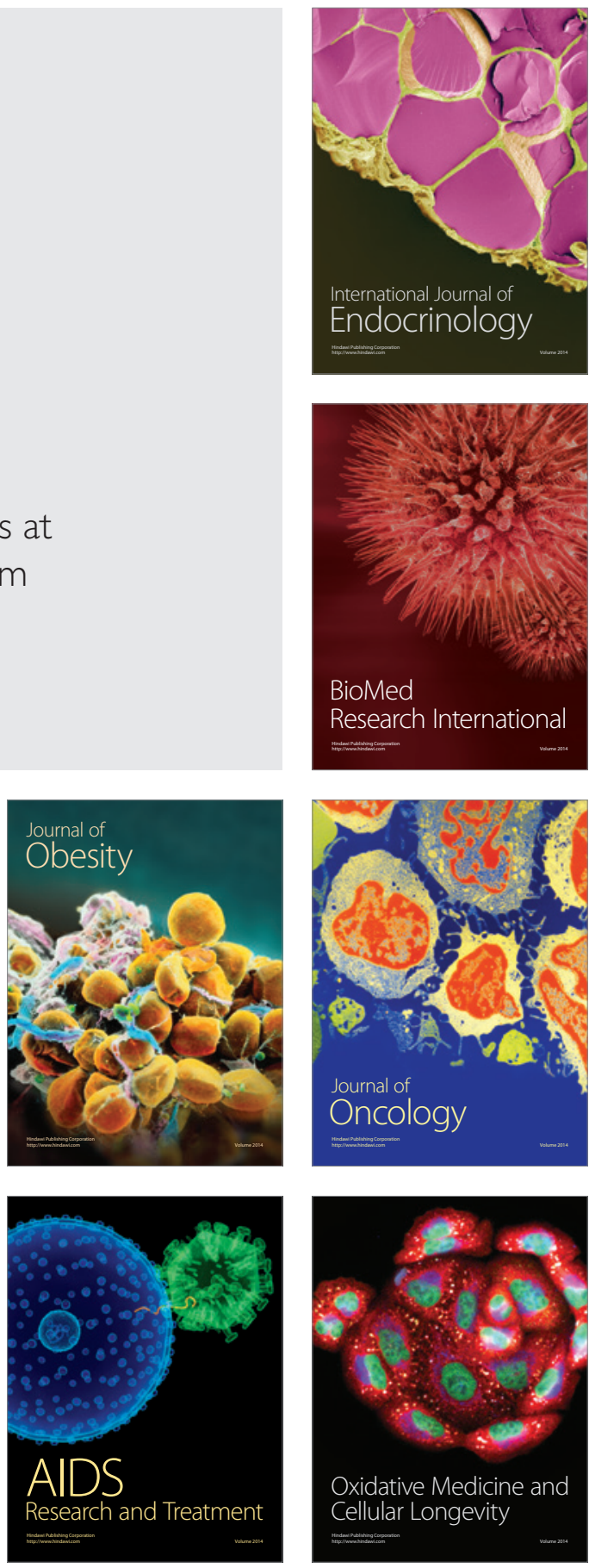